\title{
Extending a class of motion stimuli to study multiattribute texture perception
}

\author{
ANDREI GOREA \\ Université René Descartes, Paris, France and CNRS, Paris, France \\ and \\ THOMAS V. PAPATHOMAS \\ Rutgers University, New Brunswick, New Jersey
}

\begin{abstract}
We present a class of stimuli that makes it possible to study the interaction of visual attributes in forming textural patterns. These stimuli are obtained in a simple manner from a class of motion stimuli that we described earlier (Papathomas \& Gorea, 1988). The main advantages of the texture stimuli presented in this paper are: (1) each attribute can be arranged simultaneously with, but independently of, other attributes, (2) an arbitrary number of attributes can be used, (3) the interaction of attributes can be studied systematically, (4) direct comparison of two attributes is possible with stimuli in which the two are arranged to form competing patterns, and (5) because of the similarity to the motion stimuli, the relationship between texture and motion mechanisms can be investigated.
\end{abstract}

A standard method for portraying unidimensional apparent motion (AM) is to employ the $x$ - $t$ plane (Adelson $\&$ Bergen, 1985). In this method, the frame-rows that are displayed in temporal sequence to produce AM are shown with frame 0 at the top and frame $i+1$ displayed just below frame $i$. In this manner, time is mapped onto the vertical axis with its value increasing downward, enabling us to portray a motion sequence as a two-dimensional pattern. Not surprisingly, some complex $x$ - $t$ plane arrangements produce strong impressions of two-dimensional textures (see, e.g., Figures 1 and 4 of Chubb \& Sperling, 1988). As a result, we modified our class of multiattribute motion stimuli (Papathomas \& Gorea, 1988) to study the role of visual attributes in textural grouping (Beck, 1966, 1982; Callaghan, 1989; Callaghan, Lasaga, \& Garner, 1986; Garner \& Feldoldy, 1970; Nothdurft, 1985; Olson \& Attneave, 1970). In this paper, we follow closely the notation and exposition of our earlier paper on motion stimuli (Papathomas \& Gorea, 1988).

\section{MULTIATTRIBUTE TEXTURE STIMULI}

\section{Generic Form}

The generic texture stimulus is illustrated in Figure 1, in which the $x$ - and $y$-axes represent the principal spatial variables, discretized by the indices $j$ and $i$, respectively. As shown in Figure 1, texture elements (textels) occupy discrete positions on the $x-y$ plane. An element is present

Correspondence should be addressed to Andrei Gorea, Université René Descartes and CNRS, Laboratoire de Psychologie Expérimentale, 28 rue Serpente, 75006 Paris, France, or to Thomas V. Papathomas, Laboratory of Vision Research, Rutgers University, 41 Gordon RoadKilmer Campus, New Brunswick, NJ 08903. only in positions in which the sum $i+j$ of the discretized spatial variables is even, resulting in a layered-brick pattern. The width of each textel block is $p$ and the separation between blocks is $q$, resulting in an interelement distance $x_{0}$.

If one ignores the labels of the textels in Figure 1, then the microelements can be grouped in the same way along the two main diagonals (labeled $d_{B L}$ and $d_{A R}$ ), because $\Delta x$ is exactly one-half of $x_{0}$. This results in an equiprobable, ambiguous grouping along the main diagonals. One can break this ambiguity by arranging, or matching, an attribute $A$ coherently so that its values (deroted by $A_{0}$, $A_{1}$, and $A_{2}$ ) favor a textural grouping along the top-left to bottom-right, or the negatively sloped, diagonal $d_{A R}$. For example, if $A$ is color, then $A_{0}, A_{1}$, and $A_{2}$ can be red, green, and blue. Independently, attribute $B$ can be arranged coherently in space to produce a grouping that favors the positively sloped diagonal $d_{B L}$, as shown in Figure 1. There are two other basic ways in which an attribute can be arranged:

1. Multivalued ambiguous arrangement-here the values of the attribute are arranged to produce an ambiguous configuration. Two extreme subcases, which can be seen as end points of a continuum, are: (a) cyclic arrangement-this is illustrated by attribute $C$ on Figure 1 . Here, the values of $C$ cycle among the three levels, $C_{0} C_{2} C_{1} C_{0} C_{2} C_{1} \ldots$ and $C_{0} C_{1} C_{2} C_{0} C_{1} C_{2} \ldots$, along the negatively and positively sloped diagonals, respectively; and (b) random arrangement-in this case, the values of the attribute, say $D$, are assigned at random to each textel.

2. Single-valued, or constant arrangement-here, the value of the attribute, say $E$, is kept fixed at $E=E_{0}$ throughout the image. 


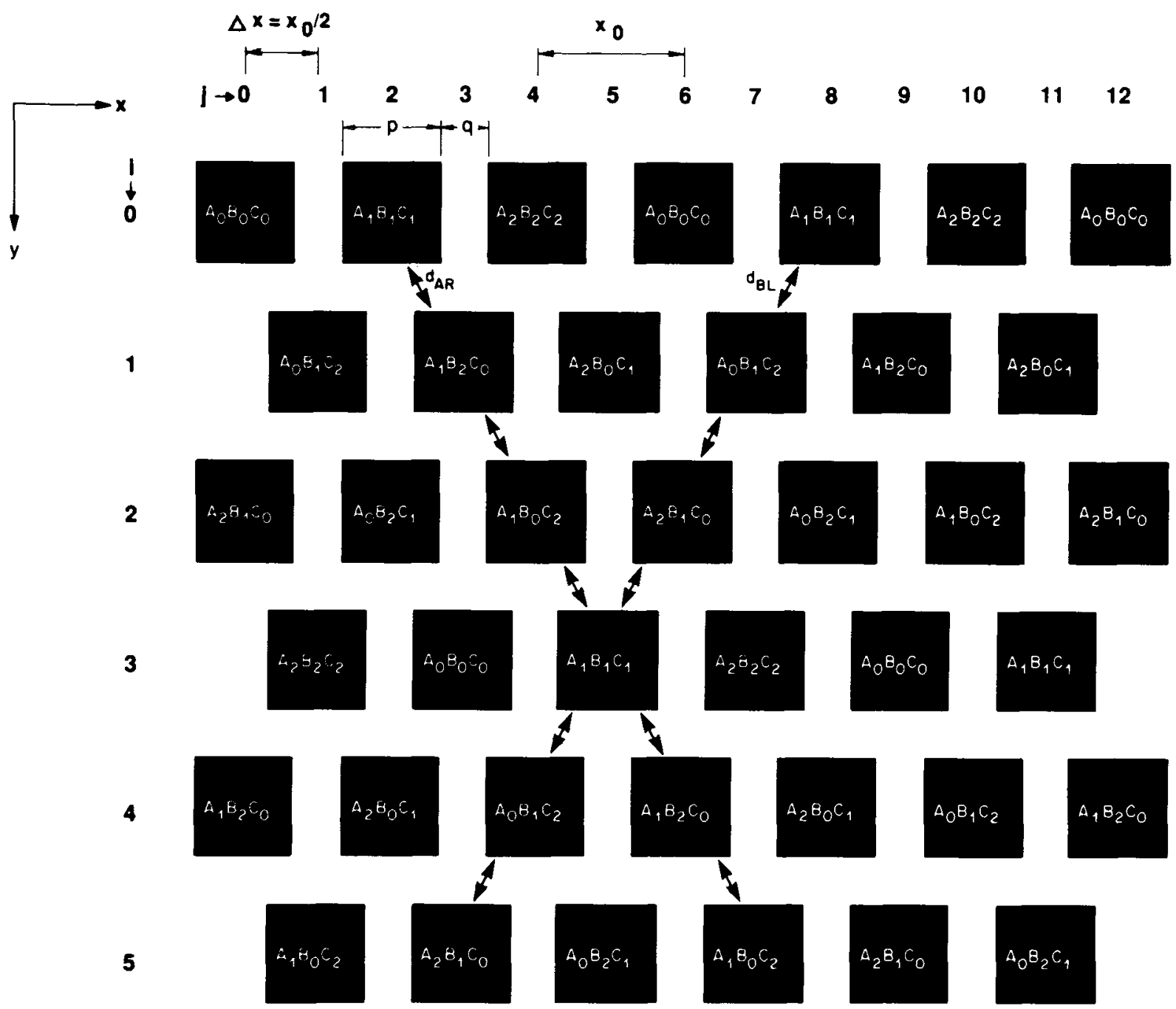

Figure 1. A schematic diagram showing the generic form of the texture stimuli. This figure is a modified version of Figure $2 \mathrm{a}$ in Papathomas and Gorea (1988). Each element is characterized by several attributes, three of which $(A, B, C)$ are shown.

Notice that each of the three arrangements just described does not favor textural grouping along either the positively or the negatively sloped diagonal, which produces ambiguous results.

\section{A Specific Example}

To illustrate the spatial arrangements and to establish our notation, we present several members of this class of stimuli in Figure 2. For simplicity, only two attributes are employed: $O=$ orientation, with $O_{0}=0^{\circ}$ and $O_{1}=$ $90^{\circ}$, and $P=$ luminance-polarity, or simply polarity, with $P_{0}=$ black and $P_{1}=$ white. Figure 2 serves only as a schematic diagram; in the actual stimuli, the background is medium gray, on which the black or white elements are displayed.

In Figure 2a, $P$ is matched to produce a grouping along the positive diagonal, whereas $O$ is held fixed (constant arrangement for $O$ ). We term this matching of $P$ within $O$, and we abbreviate it as $P_{w} O$. The converse of this arrangement, $O w P$, is shown in Figure 2b, in which $O$ is matched coherently, whereas $P$ is constant at $P_{0}$. In Figure 2c, $P$ is matched coherently, but $O$ has a cyclic arrangement. Thus, $P$ is matched across $O$; we denote this condition as $P \times O$. The converse of this arrangement, $O \times P$, is shown in Figure 2d. Of course, both attributes can be matched coherently to produce grouping along the same diagonal, as shown in Figure 2e (concurrent matching); we term this arrangement $P$ plus $O(P+O)$. Finally, the two attributes can be arranged to produce coherent groupings along different diagonals. For example, in Figure $2 f$, polarity and orientation are spatially matched to produce groupings along the positive and negative diagonals, respectively; we call this arrangement competitive matching of $P$ against $O(P \rightarrow O)$. Of course, additional patterns can be obtained by employing the random arrangement. In addition, one can randomly "jitter' the position of each textel to produce aperiodic stochastic textures. 
MOTION STIMULI AND MULTIATTRIBUTE TEXTURE PERCEPTION

7

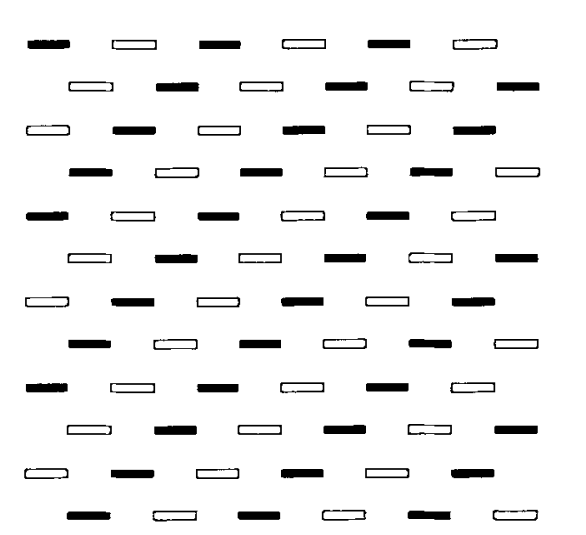

a

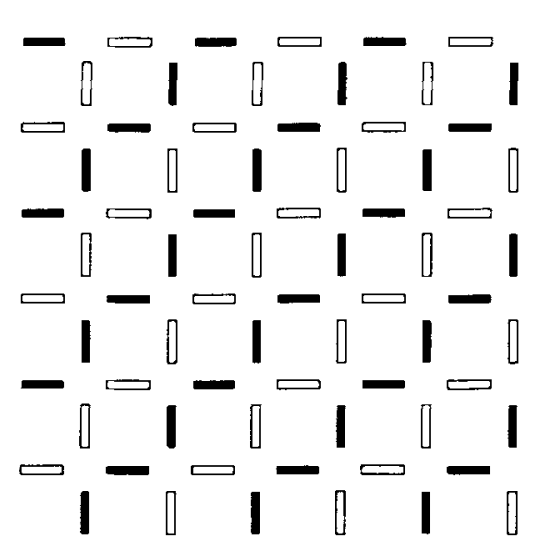

C

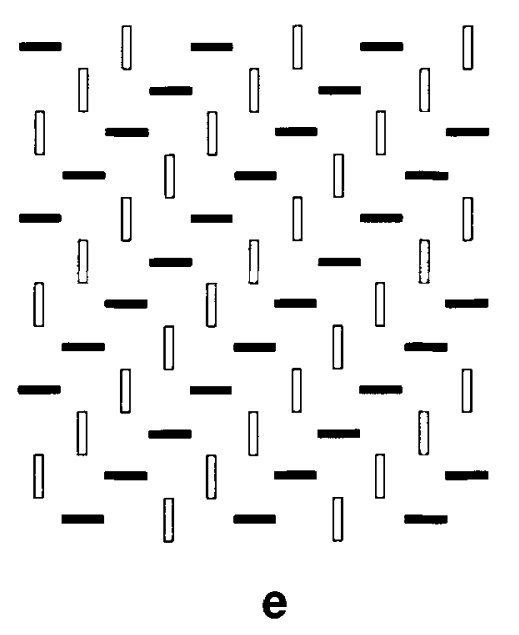

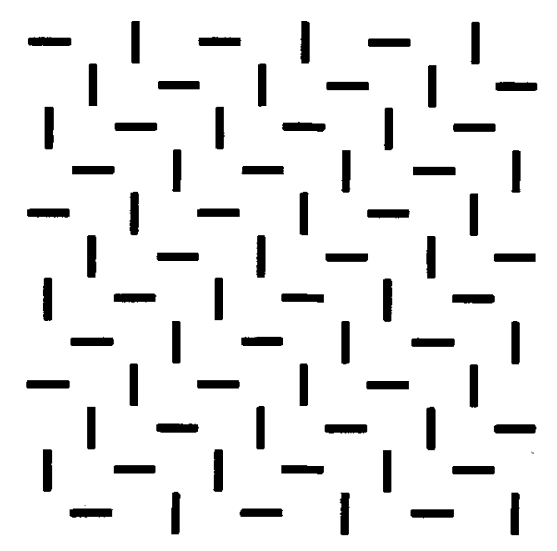

b

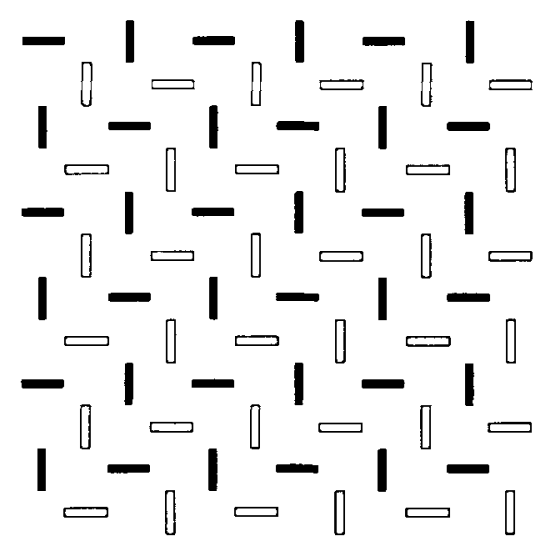

d

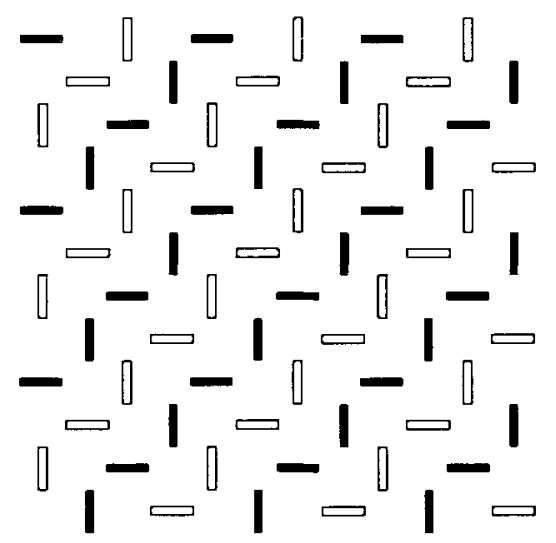

f

Figure 2. Six spatial arrangements of textels, using the attributes of $P=$ polarity and $O=$ orientation: (a) $P$ within $O(P$ w $O$ ), (b) $O$ w $P$, (c) $P$ across $O(P \times O)$, (d) $O \times P$, (e) $P$ plus $O(P+O)$, and (f) $P$ against $O$ $(P \leftrightarrow O)$. 


\section{PROPERTIES AND ADVANTAGES OF THE STIMULI}

Some of the desirable properties of this class of stimuli have been discussed in the previous sections. Below, we briefly repeat some of these characteristics and present a brief summary of additional properties and advantages: (1) As we saw, the main novel feature is that each attribute can be arranged independently of all the others; (2) an arbitrary number of attributes can be used simultaneously; (3) the interaction of visual attributes in textural pattern formation can be studied systematically; (4) if two attributes $A$ and $B$ are arranged competitively $(A \leftrightarrow B)$, as shown in Figure $2 \mathrm{f}$, one can directly compare their strength in forming textural groupings by designing experiments to find out which of the two dominates the other. The stimuli of Garner and Feldoldy (1970) also allow one to study how well an attribute groups within and across another attribute, but these stimuli are not suitable for comparing two attributes directly; and (5) since these stimuli are derived from the motion stimuli, it is possible to employ them for studying similarities and differences between motion and texture perception. As an example, we have used the stimuli of Figure 2 for studying the role of polarity and orientation in motion (Gorea \& Papathomas, 1988) and in texture grouping (Papathomas, Gorea, \& Julesz, 1990). In both the motion and texture domains, polarity was able to elicit a strong response both within and across orientation (Figures $2 \mathrm{a}$ and $2 \mathrm{c}$, respectively). By contrast, orientation elicited a strong response within polarity (Figure $2 b$ ) but a very weak response across polarity (Figure $2 \mathrm{~d}$ ). We also obtained similar results with color and orientation (Gorea \& Papathomas, 1989, in press), pointing to striking similarities between motion and texture mechanisms.

\section{CONCLUSION}

The class of stimuli presented here enables vision researchers to study the interaction of visual attributes in textural grouping. These stimuli enjoy several advantages, most of which stem from the fact that each attribute can be arranged in two-dimensional space independently of the rest of the attributes. Moreover, since these stimuli are closely related to a class of apparent motion stimuli, they open up new possibilities for investigating similarities and differences between perceptual mechanisms in motion and texture processing.

\section{REFERENCES}

ADELSON, E. H., \& BERGEN, J. R. (1985). Spatiotemporal energy models for the perception of motion. Joumal of the Optical Society of America, A2, 284-299.

BECK, J. (1966). Perceptual grouping produced by change in orientation and shape. Science, 154, 538-540.

BECK, J. (1982). Textural segmentation. In J. Beck (Ed.), Organization and representation in perception. Hillsdale, NJ: Erlbaum.

Callaghan, T. C. (1989). Interference and dominance in texture segregation: Hue, geometric form, and line orientation. Perception \& Psychophysics, 46, 299-311.

Callaghan, T. C., Lasaga, M. I., \& Garner, W. R. (1986). Visual texture segregation based on orientation and hue. Perception \& Psychophysics, 39, 32-38.

ChubB, C., Sperling, G. (1988). Drift-balanced random stimuli: A general basis for studying non-Fourier motion perception. Journal of the Optical Society of America, A5, 1986-2006.

GARNER, W. R., FELDOLDY, G. L. (1970). Integrality of stimulus dimensions in various types of information processing. Cognitive Psychology, 1, 225-241.

Gorea, A., \& Papathomas, T. V. (1988). The concept of "veto" attributes in motion perception. Investigative Ophthalmology \& Visual Science, 29, 265.

Gorea, A., \&APathomas, T. V. (1989). Motion processing by chromatic and achromatic pathways. Journal of the Optical Society of America, A6, 590-602.

Gorea, A., \& Papathomas, T. V. (in press). Texture segregation by chromatic and achromatic visual pathways: An analogy with motion processing. Journal of the Optical Society of America.

NoTHDURFT, H. C. (1985). Sensitivity for structure gradient in texture discrimination tasks. Vision Research, 25, 1957-1988.

Olson, R. K., \& ATTNEAVE, F. (1970). What variables produce similarity grouping? American Joumal of Psychology, 83, 1-21.

Papathomas, T. V., Gorea, A. (1988). Simultaneous motion perception along multiple attributes: A new class of stimuli. Behavior Research Methods, Instruments, \& Computers, 20, 528-536.

Papathomas, T. V., Gorea, A., \& Julesz, B. (1990). Juxtaposition of orientation, luminance and polarity in perceptual grouping. Investigative Ophthalmology \& Visual Science, 31, 105.

(Manuscript received September 17, 1990; revision accepted for publication November 6, 1990.) 